\title{
El contexto académico de estudiantes universitarios en condición de rezago por reprobación
}

Olga-Lidia Murillo-García y Edna Luna-Serrano

\section{RESUMEN}

El propósito de este estudio fue identificar el contexto académico de estudiantes en condición de rezago por reprobación de una universidad pública mexicana. Se contó con información de 62174 estudiantes inscritos en 77 programas de licenciatura. La fuente de información fue el Sistema Integral de Información Institucional; la unidad de análisis fue el indicador rezago por reprobación, en particular el rezago con tres o más asignaturas reprobadas (N3); se realizaron análisis descriptivos. Los resultados mostraron que la mayor proporción de estudiantes en condición de rezago N3 se encuentra en programas del área de Ingeniería y Tecnología, seguidos del área de Ciencias Naturales y Exactas, y en el Tronco Común que se imparte durante el primer año universitario en diversas áreas.

Palabras clave: rendimiento académico, rezago educativo, trayectoria escolar, reprobación, educación superior, México. 


\section{O contexto acadêmico de estudantes universitários em condição de atraso por reprovação}

\section{RESUMO}

O propósito deste estudo foi identificar o contexto acadêmico de estudantes em condição de atraso por reprovação de uma universidade pública mexicana. Contamos com informação de 62174 estudantes inscritos em 77 programas de licenciatura. A fonte de informação foi o "Sistema Integral de Información Institucional"; a unidade de análise foi o indicador atraso por reprovação, em particular o atraso com três ou más matérias reprovadas (N3); se realizaram análises descritivos. Os resultados mostraram que a maior proporção de estudantes em condição de atraso N3 se encontram em programas da área de Engenharia e Tecnologia, seguidos da área de Ciências Naturais e Exatas, e no Tronco Comum que se ministra durante o primeiro ano universitário em diversas áreas.

Palavras chave: rendimento acadêmico, atraso educativo, trajetória escolar, reprovação, educação superior, México.

\section{The academic context of failing college students}

\section{ABSTRACT}

The purpose of this study was to identify the academic context of failing students enrolled in a Mexican public university. Information was available about 62,174 students enrolled in 77 undergraduate programs. The source of information was the Integral System of Institutional Information; the unit of analysis was the indicator of failure, in particular the failure of three or more subjects (N3); descriptive analyses were made. The results showed that the greatest proportion of students in condition of N3 lagging can be observed in programs that belong to the area of Engineering and Technology, followed by the area of Natural and Exact Sciences, and in the core curriculum that is taught during the first university year in diverse areas.

Key words: academic performance, educational gap, school career, failure, higher education, Mexico. 


\section{Introducción}

El rendimiento académico ha sido interés de instituciones y de gobiernos debido a sus efectos en el desarrollo social y económico. Comprenderlo precisa el análisis de sus formas de expresión, en términos de deserción, rezago estudiantil y bajos índices de eficacia terminal, considerados componentes del fracaso escolar (Alvarado et al., 2014). Las experiencias de fracaso escolar en los individuos constituyen una privación del desarrollo de capacidades cognitivas, personales y sociales, que limitan oportunidades a bienes materiales y simbólicos, restringen las aspiraciones de los individuos y las posibilidades reales de satisfacerlas (Eroles e Hirmas, 2009). Así, el fracaso escolar puede entenderse como una condición de exclusión y marginación social (Ruíz et al., 2018).

En las últimas cuatro décadas en Iberoamérica, la matrícula en educación superior (ES) se ha incrementado más de diez veces, como consecuencia del crecimiento en la tasa de graduación en educación secundaria, diversificación en la oferta de las instituciones de educación superior (IES), programas de apoyo económico y las demandas sociales por acceder a la ES (Brunner y Miranda, 2016). A pesar de este incremento, aún se carece de una educación de calidad para todos, en la que se brinden oportunidades equitativas a los que se encuentran en condición de desventaja (Blanco, 2014; UNESCO, 2015).

En México, durante el periodo de 2016-2017, el sistema de ES registró 3429566 estudiantes. De acuerdo con las proyecciones de la Secretaría de Educación Pública (SEP, 2017), se estimó un 6.8\% de abandono escolar para dicho periodo, equivalente a 233210 estudiantes. Por otro lado, a finales del ciclo 2015 la tasa de graduación fue de $24 \%$ (SEP, 2017). En este escenario, si bien se reconocen los esfuerzos realizados por incrementar la cobertura, al mismo tiempo se estima la necesidad de políticas que atiendan la permanencia y disminuyan el abandono escolar (Mendoza, 2018).

$\mathrm{Al}$ analizar la investigación educativa en torno a la reprobación en ES, se identificó que en América predominan los estudios que analizan el fenómeno de la deserción, el fracaso escolar, el riesgo académico y en menor medida la reprobación.

Con relación al fracaso escolar, una aproximación es considerarlo como el proceso que va desde la reprobación, la repetición de cursos, hasta la deserción (Román, 2013), condiciones que involucran al rendimiento académico; por ello, uno de los mayores retos para atender el fracaso escolar consiste en determinar el perfil del estudiante en condición de riesgo. En principio, ubicar donde se encuentran, reconocer sus características y las prácticas institucionales, resulta necesario para prevenir e intervenir en el ámbito educativo.

Respecto a los estudios que atienden el riesgo académico, se identificaron tres aproximaciones principales: los que analizan las variables asociadas al bajo rendimiento (García, 2014; Oliver et al., 2011); los que buscan determinar los factores de riesgo (Lara-García et al., 2014) y los que investigan la eficacia de las estrategias de intervención en la disminución del fracaso (Hatch, 2017).

Si bien la reprobación puede intervenir en distintas situaciones que comprometen la permanencia y el éxito de los estudiantes en la escuela (García, 2014; Román, 2013), su estudio se ha realizado de manera indirecta a partir de la deserción (Dzay y Narváez, 2012), o de forma particular por disciplina (Aguilar et al., 2018; Amado et al., 2014; Riego, 2013), lo que ha derivado en la falta de una definición clara y un marco teórico sólido (Ramírez y Gallur, 2017).

En este escenario, la pregunta de investigación que orientó el presente estudio fue: ¿Cuál es el contexto académico, entendido como la etapa de formación profesional, los programas educativos y las áreas de conocimiento en que se encuentran inscritos los estudiantes en condición de rezago por reprobación de la Universidad Autónoma de Baja California (UABC)? En consecuencia, el objetivo fue identificar el contexto académico de los estudiantes en condición de rezago por reprobación de una universidad pública mexicana. 
En México, la investigación ha girado en torno a dos líneas de estudio: el rezago académico y las trayectorias escolares. El rezago académico, descrito como el retraso en las inscripciones a las asignaturas programadas en los planes de estudio, se encuentra asociado a la deserción y a la baja eficacia terminal (Vera-Noriega et al., 2012); y las trayectorias escolares han permitido identificar a los estudiantes en condición regular, de repetidor y en rezago, al analizar indicadores basados en la continuidad, aprobación, rendimiento académico y eficiencia de los estudiantes en la escuela (Ortega, 2015). Estas líneas de investigación han permitido valorar la magnitud del problema, a la vez que ofrecen una mirada de las variables que intervienen en el rendimiento académico.

De manera general, se ha señalado que la reprobación y el rezago educativo son condiciones vinculadas al fracaso escolar (Mares et al., 2013). Para valorar los aprendizajes se ha recurrido a indicadores numéricos, que constituyen lo aprendido y son símbolo del rendimiento del estudiante (Garbanzo, 2007). Así, las calificaciones corresponden a la nota académica, producto de la evaluación en un curso escolar y de pruebas objetivas estandarizadas, consideradas el criterio social y legal aceptado por las instituciones educativas (Vargas y Mortero, 2016).

Hablar de rendimiento académico alude a un término polisémico que suscita múltiples expresiones y matices para dar cuenta de la trayectoria de los estudiantes en la escuela. Para describirlo se han empleado diversas definiciones conceptuales contempladas como equivalentes, por ejemplo: aprovechamiento escolar, rendimiento educativo, logro escolar, rendimiento escolar, logro académico, pues en cada una de éstas se destaca su correspondencia con el producto de lo invertido en el proceso de enseñanza-aprendizaje (Díaz, 2014). En este trabajo se retoma el concepto de rendimiento académico.

Así, la investigación del rendimiento académico en ES se ha abordado desde diversas categorías de análisis. Gran parte de los estudios son resultado de la preocupación sobre los estudiantes que fracasan, y su objeto de estudio predominante ha sido la deserción, no la reprobación. Desde esta lógica, uno de los objetivos imperiosos en ES ha sido asegurar el éxito del estudiante, representado a través del promedio de calificaciones, la persistencia y la graduación (Olani, 2009).

De esta manera, en México, el análisis sobre los estudiantes universitarios inició con los estudios que describían sus características, algunas de ellas asociadas al rendimiento académico, centrados en conocer y analizar la situación de los estudiantes, sus prácticas e integración a las instituciones educativas desde la visión de sus actores (Garay, 2003; Guzmán, 2013; Silva-Laya, 2011), en estos estudios se perfilaron sus características demográficas, prácticas escolares y culturales con un acercamiento sociológico (Garay, 2003; 2012). Posteriormente, se desarrolló hacia el análisis de los problemas, transformaciones y desafíos que enfrentan los estudiantes en ES (Guzmán, 2013; Silva-Laya, 2012).

Un segundo enfoque fueron estudios sobre trayectoria escolar que abordaron indicadores como el rezago, el abandono y la eficacia terminal (Casillas et al., 2007; Chain y Ramírez, 1997). Subrayaron que el tránsito individual depende de procesos estructurales y sociales que ponen en tensión la permanencia de los jóvenes en la escuela, debido a que las trayectorias representan un proceso dinámico, conformado por secuencias de probabilidades que se modifican con el tiempo (Chain, 2015; Miller-Flores, 2013; 2015). Los estudios sobre trayectoria escolar describen la evolución cuantitativa y formal del tránsito de los jóvenes por la institución y reconocen como relevante la interacción de los estudiantes en la misma.

De modo que las perspectivas señaladas examinaron el fenómeno del rendimiento académico centrado en el individuo como unidad de análisis, pero dieron la pauta para girar la atención hacia las acciones institucionales (Rosales, 2015; SilvaLaya, 2012). Así, la permanencia del estudiante en 
la escuela se ha relacionado con su adaptación y, desde esta perspectiva, señala la relevancia del primer año universitario como periodo que favorece el compromiso del estudiante a su permanencia, sujeto a las experiencias y acciones del estudiante en la institución (Terenzini et al., 1996; Tinto, 1999), lo que contribuyó a reconocer las acciones institucionales que fortalecen dicho compromiso y se vinculan con la retención escolar (Tinto, 2010).

Por otra parte, el estudio sobre la reprobación en ES ha sido escaso; entre las investigaciones identificadas desde la perspectiva disciplinar, predominan los estudios realizados en las ingenierías, en las que en primer lugar destacan el rol de las variables personales (deficiencias en conocimientos previos para el dominio de las asignaturas; estrategias inadecuadas para el estudio, no asistir al aula y a las asesorías) y en segundo término, variables propias del docente como la falta de preparación didáctica y pedagógica (Aguilar et al., 2018; Amado et al., 2013; Ocampo et al., 2010; Riego, 2013). En el área de inglés, los motivos de la reprobación fueron resultado de la falta de interés por el curso y el desconocimiento del aprendizaje autónomo (Marín et al., 2015). En medicina, la reprobación es atribuida a: la edad, ser seleccionado a la residencia, el ingreso familiar, el sexo, la repetición de años en niveles educativos previos, demora en el ingreso a la ES y la actividad realizada durante el servicio social (Fernández et al., 2017); estos estudios se han enfocado en determinar las variables que intervienen en la reprobación desde su disciplina.

En resumen, analizar el rezago por reprobación implica estudiar el rendimiento académico lo que exige considerar su naturaleza multivariada. Para su estudio se ha recurrido a diversas aproximaciones y analizado numerosas variables. Además, en términos de la política educativa, se ha discutido a favor de la permanencia y la equidad de los estudiantes en ES. De esta manera, se concibe como indispensable atender el rendimiento académico con el fin de favorecer el éxito de los estudiantes.

\section{Contexto del estudio}

Este estudio se llevó a cabo en la Universidad Autónoma de Baja California (UABC). Sus campus se ubican en tres municipios del estado en los que atiende a una matrícula aproximada de 65000 alumnos, que se estima representaban cerca del $60 \%$ de la cobertura de la matrícula estatal (UABC, 2018). Para atender a dicha población se dispone de una planta docente de 5809 profesores e investigadores, de los cuales poco menos de una cuarta parte son docentes de tiempo completo, el resto con tiempo parcial o medio tiempo (UABC, 2018).

De acuerdo con la estructura curricular de los planes de estudio de la universidad, la formación profesional se organiza en tres etapas:

1. Etapa de formación básica: periodo donde se desarrollan competencias genéricas y conocimientos generales de las diversas áreas de conocimiento; corresponde a tres semestres en la mayoría de los programas, con excepción de medicina (cinco semestres). Además, esta etapa incluye una estructura denominada tronco común (TC), en la que se imparten asignaturas comunes en los programas educativos que pertenecen a una misma área de conocimiento, por lo general durante el primer año universitario.

2. Etapa disciplinaria: ciclo intermedio en el que se adquieren competencias específicas de la profesión con énfasis en competencias de conocimiento y procedimentales de su campo ocupacional.

3. Etapa terminal: destaca el desarrollo de competencias específicas y profesionales y se fomenta la vinculación universitaria con el ámbito laboral (UABC, 2014).

En la UABC, la formación integral del estudiante se señala como atributo medular del modelo educativo, por ello se dispone de programas y servicios institucionales dirigidos al acompañamiento de los 
estudiantes en sus diferentes etapas de formación, por ejemplo, el programa de tutorías y las asesorías académicas (UABC, 2014). Además, cabe mencionar que en la UABC el ingreso de los estudiantes ocurre en dos periodos semestrales (primer periodo - enero a junio-y segundo periodo - agosto a diciembre).

\section{Método}

\section{Población objeto de estudio}

Se analizó la información del estatus académico de la población de la UABC, representada por 62174 estudiantes inscritos en 77 programas educativos de licenciatura de las siguientes áreas del conocimiento: Ciencias de la Ingeniería y la Tecnología, Ciencias Agropecuarias, Ciencias de la Salud, Ciencias Naturales y Exactas, Ciencias de la Educación y Humanidades, Ciencias Sociales y Ciencias Económicas y Administrativas. Los estudiantes corresponden a siete cohortes generacionales, que van del periodo 2013-1 a 2016-1.

La muestra se constituyó por 42847 estudiantes en condición de rezago por reprobación. El criterio para su selección obedeció a la información disponible en el Sistema Integral de Información Institucional (SIII-UABC) recuperada en el periodo 2017-1.

\section{Tipo de estudio}

Corresponde a un estudio cuantitativo, descriptivo, transversal. Descriptivo, se analizó el indicador rezago por reprobación; transversal, el análisis se efectuó en un solo momento en el tiempo pues, a pesar de que se tomaron datos de siete cohortes generacionales, no se valoraron variaciones en el estatus académico.

\section{Materiales}

Se consultó el sistema de indicadores de trayectoria y seguimiento escolar, el cual forma parte del SIII-UABC. Consiste en un conjunto de sistemas que proporciona información sobre indicadores de trayectoria (eficacia terminal, eficacia de egreso, rezago educativo, tasas de deserción, de titulación y retención) y seguimiento escolar (aprobación por docente, aprobación por asignatura y programa educativo, rendimiento escolary duración promedio de los estudios) de licenciatura y posgrado. La información disponible se organiza por campus, unidad académica, programa educativo y cohorte, y se muestra en gráficas y tablas de datos en diversos formatos: JSON, XML, CSV, TXT, SQL, MS-Word y MS-Excel.

El sistema clasifica el rezago en tres niveles: rezago nivel $1(\mathrm{~N} 1)$ indica una asignatura reprobada en ordinario y extraordinario en el mismo periodo; rezago nivel 2 (N2), dos materias reprobadas, y rezago nivel 3 (N3), tres o más asignaturas reprobadas. Así, el bajo rendimiento es entendido como rezago por reprobación, descrito como un indicador que refleja la situación escolar del estudiante y se define como irregular debido a que reprobó una o más asignaturas en ordinario y extraordinario en el mismo periodo escolar (SIII-UABC, 2017).

\section{Procedimiento}

El procedimiento se realizó en tres fases:

- Fase 1. Preparación de la base de datos. Se conformó una base de datos general a partir de 71 archivos de 62174 estudiantes. La información se recuperó al especificar al sistema la cohorte generacional y el campus de los programas educativos incluidos en el SIII-UABC. En las bases de datos se incluyó: matrícula, género, nivel de rezago (N1, N2, N3), programa educativo, unidad académica, campus y cohorte.

- Fase 2. Organización de datos. La información se organizó por categorías de variables: periodo de ingreso, programa educativo, situación escolar del estudiante (regulares y nivel de rezago) y área de conocimiento. El SIII-UABC señala que el indicador rezago por reprobación representa la situación escolar del estudiante, descrita como óptima, irregular o en rezago. La situación 
óptima expresa la condición regular del estudiante e implica acreditar todas las asignaturas cursadas durante el periodo semestral.

- Fase 3. Con el propósito de identificar los programas con mayor proporción de rezago se realizaron las siguientes acciones: primero, se obtuvieron frecuencias y porcentajes por periodo de ingreso y nivel de rezago; enseguida se calcularon los porcentajes por programa educativo y se clasificaron por nivel de rezago. Debido a que el mayor porcentaje se ubicó en N3, los análisis se centraron en este nivel. Después, con base en el porcentaje de reprobación, el conjunto de programas clasificados en N3 se organizaron en cuartiles, lo que permitió distribuir los datos en partes iguales y obtener la mediana correspondiente al punto central del grupo. Finalmente, los programas se clasificaron por área de conocimiento; debido a que la clasificación atiende a particularidades de la UABC, se consideró la propuesta de Biglan (1973) y Stoecker (1993), la cual se describirá más adelante. Los análisis se realizaron con el programa Microsoft Excel (2016).

\section{Resultados}

La información que se presenta atiende al orden en que se efectuaron los análisis. De esta manera, los resultados se organizaron por nivel de rezago, por programa educativo y por área de conocimiento.

\section{Clasificación de los estudiantes por nivel de rezago}

La tabla 1 muestra la frecuencia por periodo de ingreso y nivel de rezago. Destaca mayor frecuencia de casos por rezago en los periodos semestrales 1 (ingreso enero-junio) en comparación con los periodos semestrales 2 (ingreso agosto-diciembre). Asimismo, el N3 de rezago predomina en todas las cohortes con una mayor cantidad de casos en comparación con los niveles de rezago 1 y 2 . En la última columna de la tabla 1 se muestra la sumatoria acumulada e indica el total de estudiantes inscritos en el periodo semestral 2017-1, que comprende a 62174 estudiantes. De estos, 42847 se encuentran en condición de rezago por reprobación $(68.91 \%$ de la población). Distribuidos por niveles: 31455 (50.60\%) estudiantes se ubican en N3, $5399(8.68 \%)$ en N2 y 5993 $(9.64 \%)$ en N1.

Tabla 1. Frecuencia de nivel de rezago por cohorte a partir del periodo 2013-1 a 2016-1

\begin{tabular}{|c|c|c|c|c|c|c|c|c|c|c|c|c|c|c|c|c|c|}
\hline \multirow{2}{*}{\multicolumn{2}{|c|}{$\begin{array}{l}\text { Año } \\
\text { SEM }\end{array}$}} & \multicolumn{4}{|c|}{2013} & \multicolumn{4}{|c|}{2014} & \multicolumn{4}{|c|}{2015} & \multirow{2}{*}{\multicolumn{2}{|c|}{$\begin{array}{c}2016 \\
1\end{array}$}} & & \\
\hline & & \multicolumn{2}{|c|}{1} & \multicolumn{2}{|c|}{2} & \multicolumn{2}{|c|}{1} & \multicolumn{2}{|c|}{2} & \multicolumn{2}{|c|}{1} & \multicolumn{2}{|c|}{2} & & & \multicolumn{2}{|c|}{$\begin{array}{l}\text { Sumatoria } \\
\text { acumulada }\end{array}$} \\
\hline & & Frec. & $\%$ & Frec. & $\%$ & Frec. & $\%$ & Frec. & $\%$ & Frec. & $\%$ & Frec. & $\%$ & Frec. & $\%$ & Frec. & $\%$ \\
\hline \multirow{3}{*}{ Rezago } & N1 & 536 & 6.9 & 876 & 9.1 & 639 & 8.0 & 994 & 10.2 & 796 & 9.8 & 1131 & 11.1 & 1021 & 11.7 & 5993 & 9.64 \\
\hline & $\mathrm{N} 2$ & 549 & 7.1 & 756 & 7.0 & 605 & 7.5 & 797 & 8.1 & 753 & 9.3 & 922 & 9.0 & 1017 & 11.6 & 5399 & 8.68 \\
\hline & N3 & 5274 & 68.0 & 4844 & 50.5 & 5354 & 66.7 & 4583 & 46.8 & 4628 & 57.3 & 3663 & 35.9 & 3109 & 35.6 & 31455 & 50.60 \\
\hline \multirow{2}{*}{ Total } & REZ & 6359 & 82.0 & 6476 & 67.6 & 6598 & 82.2 & 6374 & 65.1 & 6177 & 76.4 & 5716 & 56.0 & 5147 & 58.9 & 42847 & 68.91 \\
\hline & RG & 1393 & 18.0 & 3109 & 32.4 & 1424 & 17.8 & 3417 & 34.9 & 1906 & 23.6 & 4490 & 44.0 & 3588 & 41.1 & 19327 & 31.09 \\
\hline \multicolumn{2}{|c|}{ Total insc. } & 7752 & & 9585 & & 5993 & & 9791 & & 8083 & & 10206 & & 8735 & & 62174 & 100.0 \\
\hline
\end{tabular}

Nota: Rezago nivel 1 (N1), indica que los alumnos reprobaron una materia en ordinario y extraordinario en el mismo periodo; nivel 2 (N2), que los alumnos reprobaron dos materias y nivel 3 (N3), reprobaron tres o más materias en las mismas circunstancias que N1; (REZ) Rezago; (RG) Estudiantes en condición Regular, con todas las asignaturas cursadas aprobadas; (Total insc.) el total de estudiantes inscritos durante el periodo señalado; Total corresponde al número y proporción de estudiantes por estatus académico. 


\section{Nivel de rezago por programa educativo}

Con el interés de identificar los programas con mayor proporción de rezago, se jerarquizaron de acuerdo con su porcentaje en los tres niveles. Dado el número de programas, se seleccionaron las primeras 15 posiciones en cada nivel (ver tabla 2), de los cuales, en N3 se observan dos subgrupos. Los programas del primer grupo pertenecen al área de conocimiento de la Ingeniería y la Tecnología e incluyen cinco programas educativos (Ingeniero Químico, Ingeniero Eléctrico, Licenciado en Sistemas Computacionales, Ingeniero en Computación y Químico Industrial) y tres troncos comunes: TC área de Ingeniería, TC de Ciencias Agropecuarias, TG de Arquitectura y Diseño, entre los cuales más de la mitad de su población se sitúa en nivel 3 de rezago. El segundo grupo corresponde al área de conocimiento del Ciencias Naturales y Exactas, con dos programas educativos: Ciencias Computacionales y Oceanólogía, y dos troncos comunes: Ciencias Naturales y Ciencias Naturales y Exactas. Además, en este grupo se incluyen los troncos comunes de Ciencias Económicas y Políticas; área Contable-Administrativa y área de Humanidades. Destaca que los troncos comunes (8), se encuentren entre los primeros lugares de rezago N3 (ver tabla 2).

Con relación a los resultados en N2, también sobresale el número de programas de las ingenierías (7), seguido de Ciencias Sociales (4) y Ciencias de la Salud (2), y un programa de Ciencias Naturales. En $\mathrm{N} 1$, se encuentran siete programas de Ingeniería, cuatro Económico-Administrativos, tres de Educación y Humanidades, y uno de las Ciencias Sociales.

Tabla 2.Programas educativos por nivel de rezago por reprobación de las cohortes

de 2013-1 a 2016-1

\begin{tabular}{|c|c|c|c|c|c|c|}
\hline \multirow{3}{*}{ Posición } & \multicolumn{6}{|c|}{ Nivel Rezago } \\
\hline & \multicolumn{2}{|l|}{3} & \multicolumn{2}{|l|}{2} & \multicolumn{2}{|l|}{1} \\
\hline & Programa Educativo & $\%$ & Programa Educativo & $\%$ & Programa Educativo & $\%$ \\
\hline 1 & TC. Cs. Naturales & 85.28 & TC. Área de Cs. Químicas & 17.98 & Lic. Lengua y Literatura Hispanoamérica & 16.79 \\
\hline 2 & Lic. Sistemas Computacionales & 82.61 & Ing. Biotecnólogo Agropecuario & 16.67 & Ing. Biotecnólogo Agropecuario & 16.67 \\
\hline 3 & TC. (área de ingeniería) & 82.43 & Ing. Agrónomo Zootecnista & 16.13 & Lic. Teatro & 16.33 \\
\hline 4 & TC. de Cs. Agropecuarias & 80.78 & Lic. en Cs. Ambientales & 15.89 & Ing. Agrónomo Zootecnista & 16.13 \\
\hline 5 & TC. Cs. Naturales y Exactas & 79.33 & Licenciatura en Derecho & 12.38 & Lic. Relaciones Internacionales & 15.71 \\
\hline 6 & Ing. Químico & 75.68 & Lic. en Diseño Gráfico & 12.11 & Lic. Música & 14.81 \\
\hline 7 & TC. de Cs. Económicas y Políticas & 73.64 & Lic. en Enfermería & 11.58 & Lic. Biotecnología en Acuacultura & 14.56 \\
\hline 8 & TC. Arquitectura y Diseño & 71.90 & Lic. Biotecnología en Acuacultura & 11.39 & Lic. Negocios Intern acionales & 14.34 \\
\hline 9 & Ing. Eléctrico & 68.09 & Lic. Cs. Computacionales & 11.25 & Lic. Gestión Turística & 14.29 \\
\hline 10 & Lic. Cs. Comput. & 67.50 & Lic. Derecho & 11.17 & Arquitecto & 14.21 \\
\hline 11 & TC. (Área contable-Administrativa) & 67.02 & Lic. Filosofía & 11.11 & Lic. Diseño Gráfico & 14.21 \\
\hline 12 & Ing. Computación & 65.57 & Lic. Historia & 11.04 & Lic. Mercadotecnia & 13.85 \\
\hline 13 & TC. Área de Humanidades & 63.96 & Médico & 10.98 & Ing. Agrónomo & 13.49 \\
\hline 14 & Oceanólogo & 63.64 & TC. Cs. Naturales & 10.94 & TC. Área de Cs. Químicas & 13.48 \\
\hline 15 & Químico Industrial & 63.29 & Lic. Diseño Industrial & 10.89 & Lic. Docencia de la Lengua y Literatura & 13.24 \\
\hline
\end{tabular}

(TC) Tronco Común. 


\section{Programas educativos con rezago educativo N3}

Dado que la reprobación se encontró en todos los programas, pero el mayor porcentaje se identificó en N3, los análisis se concentraron en determinar los programas con mayor proporción de rezago N3. Para organizarlos, se agruparon en cuartiles (Q). Como se muestra en la figura 1, la mayor proporción corresponde al Q4 con valores que van de 59.01 a
$85.28 \%$ de los estudiantes en condición de rezago N3, que cursan ocho troncos comunes y 11 programas educativos. El Q3 va del 48.51 a $58.82 \%$; el Q2 de 33.67 a $48.15 \%$; y el Q1 de 4.62 a $33.57 \%$ (para consultar el resto de los programas ver apéndice A). La mediana de los datos corresponde a 48.14\%, lo que indica que la mitad de los programas cuentan con aproximadamente la mitad de sus estudiantes en condición de rezago.

\section{Figura 1. Porcentaje de rezago por reprobación N3 por programa educativo}

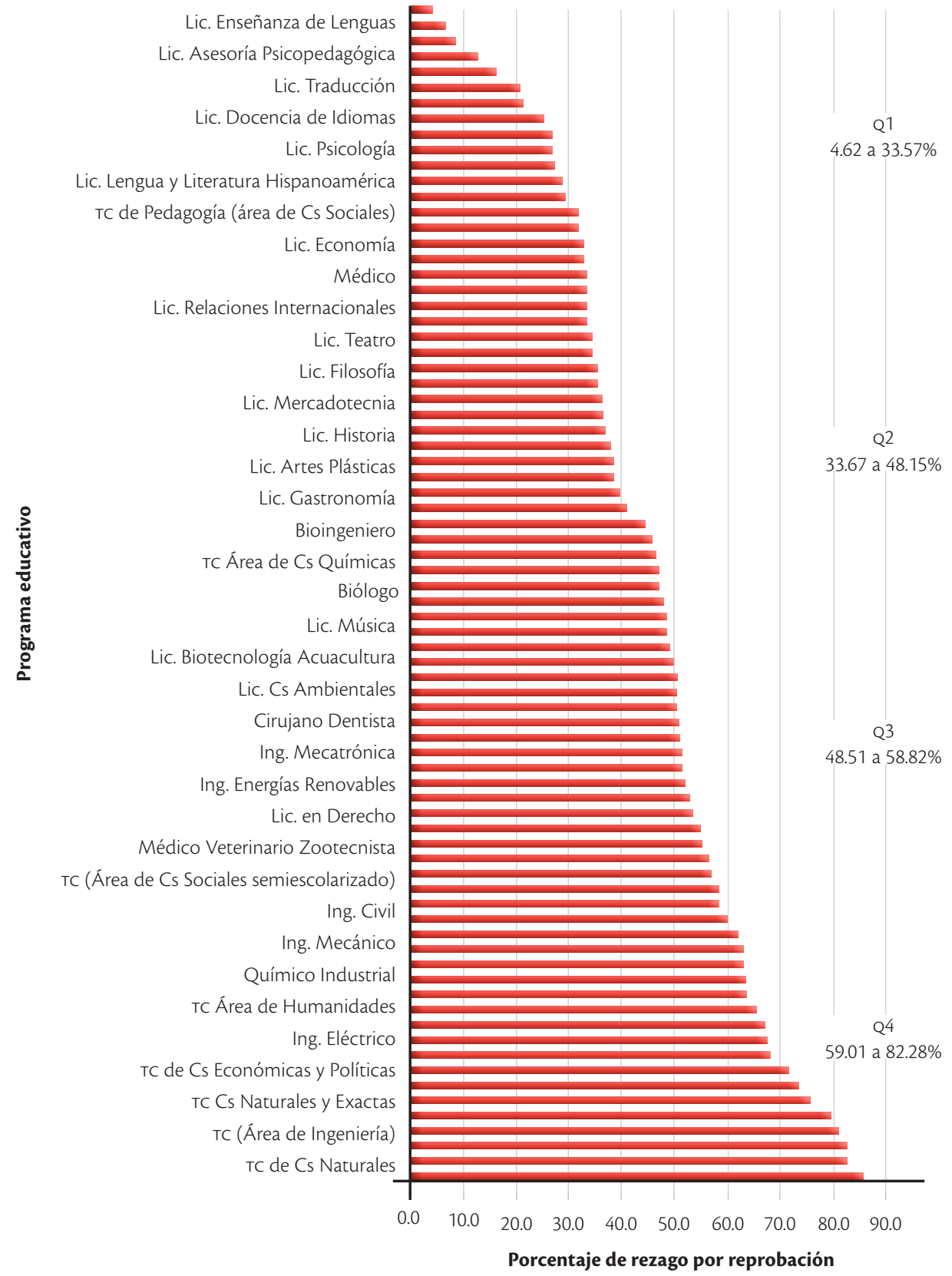




\section{Por área de conocimiento}

Las áreas de conocimiento y sus respectivos programas educativos se organizaron con base en la clasificación de Biglan (1973) y la clasificación revisada de Stoecker (1993). Para ello, se atendieron las agrupaciones por: a) categoría paradigmática, en la que los autores consideran el grado en que los estudios de la disciplina se sustentan en un paradigma o en diversos; así, los clasifican en ciencias mono-paradigmáticas (duras) o multi-paradigmáticas (suaves). b) La dimensión práctica, en la que se distingue entre las disciplinas que tienen poco uso práctico del conocimiento (puras) o aquellas que lo acentúan (prácticas). c) La dimensión concerniente a los objetos de estudio. Disciplinas implicadas en el análisis de formas orgánicas o vivas (animadas), y, por el contrario, disciplinas centradas en el estudio de cosas inorgánicas o no vivas, denominadas inanimadas. Con base en lo anterior, en la tabla 3 se muestra que el mayor número de programas con una proporción elevada de estudiantes con rezago N3 son las ciencias duras aplicadas inanimadas (11), seguido de las duras puras animadas (3).

Tabla 3. Programas educativos con mayor porcentaje de estudiantes con rezago N3 por área de conocimiento

\begin{tabular}{|c|c|c|c|c|}
\hline \multirow{2}{*}{ Categorías } & \multicolumn{2}{|c|}{ Duras } & \multicolumn{2}{|c|}{ Suaves } \\
\hline & Animada & Inanimada & Animada & Inanimada \\
\hline Puras & $\begin{array}{l}\text { TC. Cs. Naturales y Exactas } \\
\text { TC. Cs. Naturales } \\
\text { Oceanólogo }\end{array}$ & & $\begin{array}{l}\text { TC. Área de Humanidades } \\
\text { TC. Cs. Económicas y Políticas }\end{array}$ & \\
\hline Aplicadas & TC. Cs. Agropecuarias & $\begin{array}{l}\text { Ing. Civil } \\
\text { Ing. Industrial } \\
\text { Ing. Mecánica } \\
\text { Químico industrial } \\
\text { Ing. Computación } \\
\text { Lic. Cs. Computacionales } \\
\text { Ing. Eléctrico } \\
\text { TC. Arquitectura y Diseño } \\
\text { Ing. Químico } \\
\text { TC. (Área de Ingeniería) } \\
\text { Lic. Sistemas Computacionales }\end{array}$ & $\begin{array}{l}\text { TC. (Área Contable- } \\
\text { Administrativa) }\end{array}$ & \\
\hline
\end{tabular}

Nota: Clasificación de las áreas de conocimiento de acuerdo a Biglan (1973) y Stoecker (1993). TC.= Tronco común; Cs. = Ciencias; Ing.= Ingeniería 


\section{Discusión y conclusiones}

Existe consenso sobre la complejidad del rendimiento académico dado su naturaleza multicausal (Chong, 2017; Garbanzo, 2007). Estudiarlo desde diversas posturas ha permitido valorarlo en distintas manifestaciones: deserción, fracaso académico, bajo rendimiento, recursamiento, rezago educativo, persistencia, permanencia, retención, graduación, alto rendimiento, éxito académico y trayectorias escolares (Guzmán, 2013; Ortega, 2015; Silva-Laya, 2012; Vera-Noriega et al., 2012). En todas ellas se encuentra embebida la reprobación, por lo que su estudio se ha realizado de manera indirecta (García, 2014). En su mayoría, la investigación se ha centrado en el fenómeno de la deserción-retención (Munizaga et al., 2018), por lo que existen pocos estudios enfocados en la reprobación, lo que ha limitado su comprensión. Desde esta perspectiva, cobra sentido centrar la mirada en la reprobación y desarrollar acciones que permitan atender a los estudiantes que se encuentran en desventaja con el fin de promover un sistema educativo que favorezca la equidad, la permanencia y el éxito académico.

En esta investigación se encontró que la reprobación se distribuye a lo largo de todos los programas educativos. Sin embargo, la mayor concentración se ubica en los programas de Ingeniería y Tecnología, de las Ciencias Naturales y Exactas, y de las Ciencias Naturales, mismos que corresponden a las áreas de conocimiento de las ciencias duras aplicadas, ciencias duras-puras animadas e inanimadas. Congruente con estos hallazgos, se ha señalado la presencia de elevados porcentajes de reprobación en las carreras de ingenierías debido a la dificultad en la comprensión, asimilación, interpretación y aplicación de las matemáticas a situaciones concretas (Ocampo et al., 2010). Cabe señalar que los programas de las Ciencias, la Tecnología, la Ingeniería y las Matemáticas (STEM, por sus siglas en inglés), en las que se incorporan las Ciencias de la Física, la Biología, la Agricultura y las Ciencias de la Computación, son disciplinas que requieren cumplir cursos secuenciales y jerárquicos en el campo de las matemáticas, además se ha señalado que las normas de evaluación en estas áreas de conocimiento son más exigentes (Westrick, 2015). Asimismo, desde la perspectiva de las didácticas de las matemáticas, se reconoce la importancia de la acción docente (Maldonado, 2012) en el desarrollo de estrategias pedagógicas apropiadas para la enseñanza de las matemáticas, como la implementación de actividades sistemáticas que favorezcan actitudes positivas centradas en la autoeficacia en un entorno que privilegie la interacción positiva entre docentes y estudiantes (Vargas y Mortero, 2016), por lo que destaca la importancia de indagar lo que acontece en el aula.

De manera particular sobresale la relevancia del primer año universitario, debido a que en el tronco común (tramo formativo compuesto por los dos primeros semestres para la mayoría de los programas educativos) se observaron porcentajes elevados de rezago por reprobación N3 no solo en las áreas de STEM, sino que se incorporaron las ciencias Económico-Políticas, Contable-Administrativa y Humanidades. Esta situación no es ajena al escenario nacional, dado que se ha destacado la trascendencia de esa etapa debido a sus implicaciones en el compromiso estudiantil, y la integración social y académica que favorecen la permanencia del estudiante en la escuela (Tinto, 2010; Holder et al., 2016; Silva-Laya, 2011; 2012); por ello se considera ineludible reconocer institucionalmente la importancia del primer año universitario, con el fin de implementar estrategias que atiendan las necesidades particulares de esta población (Silva-Laya, 2011).

Los resultados del estudio permitieron identificar el contexto académico de estudiantes en condición de rezago por reprobación. Realizar este trabajo fue posible debido a que la UABC dispone de un sistema de indicadores, medio que facilita el acceso a información relevante e indispensable para describir y plantear problemas, lo que posibilita detectarlos 
de manera oportuna y tomar decisiones en materia educativa.

Como se ha señalado, es deseable que las instituciones desarrollen estrategias de retención que favorezcan la permanencia y el éxito académico de los educandos (Goodman y Pascarella, 2006). Entre las recomendaciones se encuentran: a) los seminarios para estudiantes de primer año (Porter y Swing, 2006) con el propósito de fomentar hábitos de estudio, administración de tiempo, y acciones que apoyen la autorregulación del aprendizaje (García, 2014; Vargas y Montero, 2016), además de atender componentes afectivos como la motivación (García-Ros et al., 2017); b) programas de tutorías (García et al., 2012); c) programas de atención al progreso académico
(Chi y Dow, 2014) o, en otras palabras, comunidades de aprendizaje que acompañen al estudiante en el tránsito por la universidad y apoyen la integración social en sintonía con la integración académica (Tinto, 1999); y d) poner en el centro de las políticas de retención el trabajo en el aula (American Federation of Teachers in Higher Education [AFT], 2011). Este estudio es un primer acercamiento a la caracterización de los estudiantes en condición de rezago por reprobación, en su expresión más general, como es el programa, área y etapa de formación en la que se encuentran; lo que permite establecer su contexto académico y que no se pierdan en la inmensidad de una universidad, para ser atendidos con el compromiso de implementar medidas acordes a sus necesidades. 


\section{Apéndice A. Programas educativos y con mayor porcentaje de rezago N3} agrupados en cuartiles

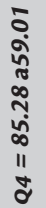

\begin{tabular}{|c|c|c|}
\hline & Programa Educativo & $\%$ \\
\hline & TC. Cs. Naturales & 85.28 \\
\hline & Lic. Sistemas Computacionales & 82.61 \\
\hline & TC. (Área de Ingeniería) & 82.43 \\
\hline & TC. Cs. Agropecuarias & 80.78 \\
\hline & TC. Cs. Naturales y Exactas & 79.33 \\
\hline & Ing. Químico & 75.68 \\
\hline & TC. Cs. Económicas y Políticas & 73.64 \\
\hline & TC. Arquitectura y Diseño & 71.90 \\
\hline & Ing. Eléctrico & 68.09 \\
\hline & Lic. Cs. Computacionales & 67.50 \\
\hline & TC. (Área Contable-Administrativa) & 67.02 \\
\hline & Ing. Computación & 65.57 \\
\hline & TC. Área de Humanidades & 63.96 \\
\hline & Oceanólogo & 63.64 \\
\hline & Químico industrial & 63.29 \\
\hline & Físico & 63.20 \\
\hline & Ing. Mecánico & 62.34 \\
\hline & Ing. Industrial & 60.43 \\
\hline & Ing. Civil & 59.01 \\
\hline & Programa Educativo & $\%$ \\
\hline & Lic. Música & 48.15 \\
\hline & Lic. Administración Pública y Cs. Políticas & 47.22 \\
\hline & Biólogo & 46.96 \\
\hline & Lic. Matemáticas Aplicadas & 46.81 \\
\hline & TC. Área de Cs. Químicas & 46.07 \\
\hline & Lic. Danza & 44.83 \\
\hline & Bioingeniería & 41.35 \\
\hline & Lic. Administración de Empresas & 40.35 \\
\hline & Lic. Gastronomía & 38.72 \\
\hline & Ing. Aeroespacial & 38.64 \\
\hline & Lic. Artes Plásticas & 38.19 \\
\hline & Arquitecto & 37.09 \\
\hline & Lic. Historia & 36.81 \\
\hline & Lic. Actividad Física y Deporte & 36.63 \\
\hline & Lic. Mercadotecnia & 35.51 \\
\hline & Ing. Agrónomo Zootecnista & 35.48 \\
\hline & Lic. Filosofía & 34.92 \\
\hline & Lic. Diseño Grafico & 34.74 \\
\hline & Lic. Teatro & 33.67 \\
\hline
\end{tabular}

\begin{tabular}{|c|c|c|}
\hline & Programa Educativo & $\%$ \\
\hline & Ing. Nanotecnología & 58.82 \\
\hline & TC. (Área Cs. Sociales Semiescolarizado) & 57.23 \\
\hline & Químico Fármaco-Biólogo & 56.63 \\
\hline & Médico Veterinario Zootecnista & 55.45 \\
\hline & TC. Pedagogía (Cs. Sociales) modalidad en línea & 55.15 \\
\hline & Licenciatura en Derecho & 53.96 \\
\hline & Ing. Electrónica & 53.2 \\
\hline & Lic. Derecho & 52.19 \\
\hline & Lic. Enfermería & 51.93 \\
\hline & Ing. Energías Renovables & 51.70 \\
\hline & Ing. Agrónomo & 51.56 \\
\hline & Ing. Mecatrónica & 51.42 \\
\hline & TC. (Área de Cs. Sociales) & 50.92 \\
\hline & Cirujano Dentista & 50.75 \\
\hline & TC. Idiomas & 50.50 \\
\hline & Lic. Cs. Ambientales & 50.47 \\
\hline & Lic. Informática & 48.8 \\
\hline & Lic. Biotecnología en Acuacultura & 48.73 \\
\hline & Lic. Sociología & 48.51 \\
\hline \multirow{21}{*}{$\begin{array}{l}\hat{\sigma} \\
\dot{+} \\
\dot{\alpha} \\
\hat{n} \\
\tilde{m} \\
m \\
11 \\
\tilde{\sigma}\end{array}$} & Programa Educativo & $\%$ \\
\hline & Lic. Gestión Turística & 33.5 \\
\hline & Lic. Relaciones Internacionales & 33.42 \\
\hline & Ing. Biotecnólogo Agropecuario & 33.33 \\
\hline & Médico & 33.37 \\
\hline & Lic. Cs. de la Comunicación & 32.86 \\
\hline & Lic. Economía & 32.09 \\
\hline & Lic. Contaduría & 31.87 \\
\hline & TC. Pedagogía (Área Cs. Sociales) & 29.66 \\
\hline & Lic. Medios Audiovisuales & 28.96 \\
\hline & Lic. Lengua y Literatura Hispanoamérica & 27.48 \\
\hline & Lic. Diseño Industrial & 27.23 \\
\hline & Lic. Psicología & 27.02 \\
\hline & Lic. Negocios Internacionales & 25.43 \\
\hline & Lic. Docencia de Idiomas & 21.51 \\
\hline & Lic. Cs. de la Educación & 20.96 \\
\hline & Lic. Traducción & 16.59 \\
\hline & Lic. Docencia de la Lengua y Literatura & 12.87 \\
\hline & Lic. Asesoría Psicopedagógica & 9.40 \\
\hline & Lic. Docencia de la Matemática & 7.07 \\
\hline & Lic. Enseñanza de lenguas & 4.62 \\
\hline
\end{tabular}




\section{Referencias}

American Federation of Teachers in Higher Education (AFT) (2011), Student success in higher education, EUA, American Federation of Teachers in Higher Education (Libro electrónico), <https://www.aft.org/sites/default/ files/studentsuccess0311.pdf $>$ [Consulta: marzo de 2019].

Aguilar, Wendolyn, Maximiliano Fuentes, Cesar Iñiguez

y Ruth Rivera (2018), "Perfiles de estudiantes asociados a las características de reprobación de las asignaturas de ciencias básicas en ingeniería”, Boletín Redipe, vol. 7, num. 8, pp. 129-145, <https://dialnet.unirioja.es/ servlet/articulo?codigo $=6523207>$ [Consulta: octubre de 2019].

Alvarado, Irma, Zaira Vega, María Luisa Cepeda y Ana Elena del Bosque (2014), "Comparación de estrategias de estudio y autorregulación en universitarios", Revista Electrónica de Investigación Educativa, vol.16, núm. 1, pp. 137-148<http://redie.uabc.mx/vol16nol/contenidoalvarado-vegaetal.html> [Consulta: enero de 2019].

Amado, María-Guadalupe, Ángel García, Reyna-Arcelia Brito, Bertha-Ivonne Sánchez y Carlos-Alfonso Sagaste (2014), “Causa de reprobación en ingeniería desde la perspectiva del académico y administradores", Ciencia y Tecnología, vol. 1, núm. 14, pp. 233-250, <https://www. palermo.edu/ingenieria/pdf2014/14/CyT_14_15. pdf> [Consulta: octubre de 2019].

Biglan, Anthony (1973), "The characteristics of subject matter in different academic areas", fournal of Applied Psychology, vol. 57, núm. 3, pp. 195-203, DOI: doi.10.1037/h0034701 [Consulta: enero de 2019].

Blanco, Rosa (2014), "Inclusión educativa en América Latina: caminos recorridos y por recorrer", en Á. Marchesi, R. Blanco, y L. Hernández, Avances y desafios de la educación inclusiva en Iberoamérica, Madrid, Organización de los Estados Iberoamericanos para la Educación, la Ciencia y la Cultura, pp. 11-36.

Brunner, Joaquín y Andrés Miranda (2016), "Informe sobre educación superior en Iberoamérica", Santiago de Chile, CINDA/Universia/Telefónica/World Bank, pp.
280, <https://cinda.cl/publicacion_archivos/educacionsuperior-en-iberoamerica-informe-2016/ [Consulta: septiembre de 2018].

Casillas, Miguel, Ragueb Chain y Nancy Jácome (2007), "Origen social de los estudiantes y trayectorias estudiantiles en la universidad veracruzana", Revista de la Educación Superior, vol. 36, núm. 2, pp. 7-29.

Chain, Ragueb y Concepción Ramírez (1997), "Trayectoria escolar: un estudio sobre la eficacia terminal en la UV", Revista de la Educación Superior, núm. 102, pp. 1-11, <http://publicaciones.anuies.mx/ pdfs/revista/Revista102_S2A2ES.pdf> [Consulta: diciembre de 2017].

Chain, Ragueb (2015), "Prólogo", en J. C. Ortega Guerrero, R. López González, y E. Alarcón Montiel (eds.), Trayectorias escolares en educación superior. Propuesta metodológica y experiencias en México, Xalapa, México, Universidad Veracruzana, pp. 9-14

Chi, Oliva y Aaron Dow (2014), "Improving balance in regression discontinuity design by matching: estimating the effect of academic probation after first year of college", en Society for Research on educational effectiveness [SREE], conferencia primavera de 2014 (Base de datos ERIC), <https://files.eric.ed.gov/fulltext/ED562761. pdf> [Consulta: enero de 2019].

Chong, Elizabeth (2017), "Factores que inciden en el rendimiento académico de los estudiantes de la Universidad Politécnica del Valle de Toluca", Revista Latinoamericana de Estudios Educativos, vol. 47, núm. 1, pp. 91-108, <http://www.redalyc.org/articulo. oa?id=27050422005> [Consulta: enero de 2019].

Díaz, Karla (2014), "Modelo explicativo del rendimiento académico en español de estudiantes de secundaria", Tesis Doctoral, Universidad Autónoma de Baja California, Baja California.

Dzay, Floricely y Manuel Narváez (2012), La deserción escolar desde la perspectiva estudiantil, México, Manda.

Eroles, Daniela y Carolina Hirmas (2009), "Lecciones aprendidas sobre experiencias educativas de segunda 
oportunidad", en Experiencias educativas de segunda oportunidad. Lecciones desde la práctica innovadora en América Latina, Santiago, Chile, OREALC-UNESCO, pp. 180234, <https://unesdoc.unesco.org/ark:/48223/ pf0000186472> [Consulta: junio de 2018].

Fernández, Miguel-Ángel, Armando Ortiz, Efrén-Raúl Ponce, Guillermo Fajardo, Irma Jiménez y Juan-José Mazón(2017), "Reprobación en estudiantes de Medicina de la Universidad Nacional Autónoma de México", Educación Médica Superior, vol. 31, num. 4, pp. 1-17, <https://www.medigraphic.com/pdfs/educacion/ cem-2017/cem1741.pdf> [Consulta: octubre de 2018].

Garay, Adrian (2012), Mis estudios y propuestas sobre los jóvenes universitarios mexicanos, México, Unión de Universidades de América Latina y el Caribe <https://www.ses. unam.mx/curso2015/pdf/28oct-DeGaray.pdf> [Consulta: septeimbre de 2018].

Garay, Adrián (2003), "El perfil de los estudiantes de nuevo ingreso de las universidades tecnológicas en México", El Cotidiano, vol. 19, núm. 122, pp. 75-85, <http://www. redalyc.org/articulo.oa? $\mathrm{id}=32512209>$ [Consulta: enero de 2018].

Garbanzo, Guiselle (2007), "Factores asociados al rendimiento académico en estudiantes universitarios, una reflexión desde la calidad de la educación superior pública", Revista Educación, vol. 31, núm. 1, pp. 43-63, <https://www.redalyc.org/pdf/440/44031103.pdf> [Consulta: enero de 2019].

García, Ramona, Omar Guevas, Javier Vales e Isidro Cruz (2012), "Impacto del Programa de Tutoría en el desempeño académico de los alumnos del Instituto Tecnológico de Sonora", Revista Electrónica de Investigación Educativa, vol. 14, núm. 1, pp. 160-121, <http:// redie.uabc.mx/vol14nol/contenido-garciaetal.html> [Consulta: marzo de 2019].

García, Ana-María (2014), "Rendimiento académico y abandono universitario. Modelo, resultados y alcances de la producción académica en la Argentina", Revista Argentina de Educación Superior, núm. 8, pp. 9-38, <https:/ / dialnet.unirioja.es/servlet/articulo? codigo $=4753763>$ [Consulta: enero de 2019].
García-Ros, Rafael, Francisco Pérez-González, Francisco Cavas-Martínezy José Tomás (2017), "Social interaction learning strategies, motivation, first-year students' experiences and permanence in university studies", Educational Psychology, vol. 38, núm. 4, pp. 451-469, DOI: 10.1080/01443410.2017.1394448 [Consulta: enero de 2019].

Goodman, Kathleen y Ernest Pascarella (2006), "Firstyear seminars increase persistence and retention", Peer Review, vol. 8, núm. 3, pp. 26-29, <https://www. researchgate.net/publication/327802482> [Consulta: marzo de 2019].

Guzmán, Carlota (2013), Los estudiantes y la universidad. Integración, experiencias e identidades, México, ANUIES.

Hatch, Deryl (2017), "The structure of student engagement in community college student success programs: A quantitative activity systems analysis", American Educational Research Association Open, vol. 3, núm. 4, pp. 1-14, DOI: doi.org/10.1177/2332858417732744 [Consulta: enero de 2018].

Holder, Tyson, Sean Chism, Theresa Keuss y Natissia Small (2016), "Retention and persistence in higher education: an exploratory study of risk factors and milestones impacting second semester retention of freshmen students", Tesis Doctoral, University of Missouri-St. Louis, <https://irl.umsl.edu/dissertation> [Consulta: enero de 2019].

Lara-García, Baudelio, Aarón González-Palacios, María de los Ángeles González-Álvarez y María Guadalupe Martínez-González (2014), "Fracaso escolar: conceptualización y perspectivas de estudio", Revista de Educación y Desarrollo, núm. 30, pp. 71-83, <http://www. cucs.udg.mx/revistas/edu_desarrollo/anteriores/30/30_ Lara.pdf> [Consulta: julio de 2019].

Maldonado, Anahy (2012), "Identificación de factores que intervienen en la reprobación del curso de matemáticas básicas de a FGFM de la BUAP”, Tesis de Licenciatura, Puebla, Benemérita Universidad Autónoma de Puebla, <https://www.fcfm.buap.mx/assets/docs/docencia/ tesis/matematicas / AnahyMaldonadoGarcia.pdf> [Consulta: marzo de 2019]. 
Mares, Guadalupe, Luis González, Olga Rivas, Héctor Rocha, Elena Rueda, Luis Enrique Rojas, Daniela Cruz y Roberto López (2013), "Trayectorias discontinuas en educación superior: el caso de alumnos de la carrera de psicologia de iztacala, México", Revista Mexicana de Investigación en Psicología, vol. 5, núm. 1, pp. 71-81, <http://www.medigraphic.com/pdfs/revmexinvpsi/ mip-2013/mip 131f.pdf> [Consulta: enero de 2019].

Marín, Claudia-Isabel, Emily Librado y María-Eugenia Alarcón (2015), "Estudiantes universitarios en situación de examen de última oportunidad de Inglés I", Revista electrónica Actualidades Investigativas en Educación, vol. 15, núm. 1, pp. 1-20, <https://www.redalyc.org/articulo. oa?id $=44733027010>$ [Consulta: octubre de 2019].

Mendoza, Javier (2018), "Situación y retos de la cobertura del sistema educativo nacional", Perfiles educativos, vol. 40 (número especial), pp. 11-52, <http://www.iisue. unam.mx/perfiles/articulo/2018-e-situacion-y-retosde-la-cobertura-del-sistema-educativo-nacional.pdf $>$ [Consulta: enero de 2019].

Miller-Flores, Dinorah (2013), "El estudio de las trayectorias escolares en México. un aporte para el nuevo milenio", en Saucedo, C., Estudiantes, maestros y académicos en la investigación educativa. Tendencias, aportes y debates 2011-2011, México, ANUIES.

Miller-Flores, Dinorah (2015), "Exploración del vínculo entre trayectorias escolares y marcos normativos en la Universidad Autónoma Metropolitana", Revista Iberoamericana de Educación Superior, vol. 6, núm. 17, pp. 21-37 <https://www.sciencedirect.com/science/ article/pii/S2007287215000335> [Consulta: septiembre de 2018].

Munizaga, Felipe, M. Beatriz Cifuentes y Andrés Beltrán (2018), "Retención y abandono estudiantil en la educación superior universitaria en América Latina y el Caribe: una revisión sistemática", Archivos Analíticos de Políticas Educativas, vol. 26, núm. 61, pp. 1-36, DOI: doi. org/10.14507/epaa.26.3348 [Consulta: enero de 2019].

Ocampo, Juan, Miguel Martínez, Maximiliano Fuentes y Jorge Zatarain (2010), "Reprobación y deserción en la facultad de ingeniería Mexicali de la Universidad
Autónoma de Baja California", <https://www. repositoriodigital.ipn.mx/handle/123456789/3653> [Consulta: marzo de 2019].

Olani, Aboma (2009), "Predicting first year university students academic success", Electronic Fournal of Research in Educational Psychology, vol. 7, núm. 3, pp. 1053-172, <http://ojs.ual.es/ojs/index.php/EJREP/article/ view/1351/1501> [Consulta: noviembre de 2018].

Oliver, María, Griselda Eimer, Nancy Bálsamo y Mónica Crivello (2011), "Permanencia y abandono en química general en las carreras de ingeniería de la Universidad Tecnológica Nacional-Facultad Regional Córdoba (UTC/FRC), Argentina", Avances en Ciencias e Ingeniería, vol. 2, núm. 2, pp. 117-129, <https://www.redalyc. org/articulo.oa? id=323627682011> [Consulta: septiembre de 2019].

Ortega, Juan-Carlos (2015), "Surgimiento de la propuesta del estudio de las trayectorias escolares en la universidad veracruzana”, en J. C. Ortega, R. López y E. Alarcón (coord.), Trayectorias escolares en educación superior. Propuesta metodológica y experiencias en México, México, Universidad Veracruzana, pp. 23-42.

Porter, Stephen y Randy Swing (2006), "Understanding how first-year seminars affect persistence", Research in Higher Education, vol. 47, núm. 1, pp. 89-109, DOI: doi. 10.1007/s11162-005-8153-6 [Consulta: enero de 2019].

Ramírez, Leticia-Nayeli y Santiago Gallur (2017), "La perspectiva socio-cultural como modelo teórico de análisis de la reprobación académica en educación superior", Revista Científico Pedagógica Atenas, vol. 2, núm. 38, pp. 1-17, <https://www.aacademica.org/leticia. ramirez/10> [Consulta: octubre de 2019].

Riego, María (2013), "Factores académicos que explican la reprobación en Cálculo diferencial”, Conciencia Tecnológica, núm. 46, pp. 29-35, <http://www.redalyc. org/articulo.oa?id=94429298006> [Consulta: julio de 2018].

Román, Marcela (2013), "Factores asociados al abandono y la deserción escolar en América Latina: una mirada en conjunto", REICE, Revista Iberoamericana sobre Calidad, 
Eficacia y Cambio en Educación, vol. 11, núm. 2, pp. 3359, <http://www.rinace.net/reice/numeros/arts/ vol1 1 num2/art2.pdf> [Consulta: septiembre de 2017].

Rosales, Oliva (2015), "Estudio de las condiciones contextuales que promueven la permanencia, tránsito y egreso de los estudiantes en la universidad", Tesis de Maestría, Ensenada, Universidad Autónoma de Baja California.

Ruíz, Ana, María Palma y Juan Álvarez (2018), “Jóvenes NiNi. Nuevas trayectorias hacia la exclusión social", Revista Internacional de Trabajo Social y Ciencias Sociales, núm. 15, pp. 39-49, DOI: doi.org/10.5944/ comunitania.15.2 [Consulta: noviembre de 2018].

Secretaria de Educación Pública (SEP) (2017), Principales cifras del sistema educativo nacional 2016-2017, México, Dirección General de Planeación, Programación y Estadística Educativa, SEP, <https://www.planeacion. sep.gob.mx/Doc/estadistica_e_indicadores/ principales_cifras/principales_cifras_2016_2017_ bolsillo.pdf> [Consulta: enero de 2019].

SII-UABC (2017), Indicadores de trayectoria y seguimiento escolar (Base de datos Sistema Integral de Información Institucional), Mexicali, B.C., UABC, <http://siii.uabc.mx/> [Consulta: enero de 2017].

Silva-Laya, Marisol (2011), "El primer año universitario. Un tramo crítico para el éxito académico", Perfiles Educativos, vol. 33, pp. 102-114, <http://www.redalyc. org/articulo.oa?id=13221258010> [Consulta: junio de 2018].

Silva-Laya, Marisol (2012), "Equidad en educación superior en México: la necesidad de un nuevo concepto y nuevas políticas", Education Policy Analysis Archives, vol. 20, pp. 1-27, <http://www.redalyc.org/articulo. oa? id=275022797004> [Consulta: enero de 2019].

Stoecker, Judith. L. (1993), "The Biglan classification revised", Research in Higher Education, vol. 34, núm. 4, pp. 451-464, <https://link.springer.com/content/ pdf/10.1007/BF00991854.pdf> [Consulta: enero de 2019].

Terenzini, Patrick T., Leonard Springer, Patricia M. Yaeger, Ernest T. Pascarella y Amaury Nora (1996),
"First-generation college students: characteristics, experiences, and cognitive development", Research in Higher Education, vol. 37, núm. 1, pp. 1-22, DOI: doi. org/10.1007/BF01680039 [Consulta: junio de 2018].

Tinto, Vincent (2010), "From theory to action: exploring the institutional conditions for student retention", en J. C. Smart (ed.), Higher Education: Handbook of Theory and Research, vol. 25, pp. 51-89, Springer, DOI: 10.1007/978-90-481-8598-6_2.

Tinto, Vincent (1999), "Taking retention seriously: rethinking the first year of college", National Academic Advising Association fournal (NACADA), vol. 9, núm. 2, pp. 5-9, DOI:doi/pdf/10.12930/0271-9517-19.2.5 [Consulta: noviembre de 2018].

UNESCO (2015), Educacion 2030 Declaración de Incheon Hacia una educación inclusiva y equitativa de calidady un aprendizaje a los largo de la vida para todos, Icheon, Republica de Corea, UNESCO (Biblioteca Digital UNESCDOC), <https:// unesdoc.unesco.org/ark:/48223/pf0000245656_spa> [Consulta: septiembre de 2018].

Universidad Autónoma de Baja California (UABC) (2018), Informe de resultados institucionales. Gestión rectoral 2015-2019, <http://www.ens.uabc.mx/documentos/PDCE\%20 2015-2019.pdf> [Consulta: diciembre de 2018].

UABC (2014), Modelo educativo de la UABC, Mexicali, B.C., UABC.

Vargas, Marcia y Eiliana Montero (2016), "Factores que determinan el rendimiento académico en matemáticas en el contexto de una universidad tecnológica: aplicación de un modelo de ecuaciones estructurales", Universitas Psychologica, vol. 15, núm. 4, DOI: 10.11144/ Javeriana.upsy15-4.fdra [Consulta: marzo de 2019].

Vera-Noriega, José-Ángel, Dora-Yolanda Ramos-Estrada, Mirsha-Alicia Sotelo-Castillo, Sonia EcheverríaCastro, Dulce-María Serrano-Encinas y Javier-José Vales-García (2012), "Factores asociados al rezago en estudiantes de una institución de educación superior en México", Revista Iberoamericana de Educación Superior, vol. 3, núm. 7, pp. 41-56, DOI: https://doi.org/10.22201/ iisue.20072872e.2012.7.64 [Consulta: marzo de 2019]. Westrick, Paul (2015), "Differential grading: meta-analyses 
of STEM and Non-STEM fields, gender, and institutional admission selectivity", ACT Research Report Series, vol. 7, pp.1-40 <https://files.eric.ed.gov/fulltext/ED563775. pdf $>$ [Consulta: marzo de 2019].

\section{Cómo citar este artículo:}

Murillo-García, Olga-Lidia y Edna Luna-Serrano (2021), "El contexto académico de estudiantes universitarios en condición de rezago por reprobación”, Revista Iberoamericana de Educación Superior (RIES), vol. XII, núm. 33, pp. 58-75, DOI: https://doi.org/10.22201/ iisue.20072872e.2021.33.858 [Consulta: fecha de última consulta]. 